\title{
Wireless Novel Nanotechnology in Neuromodulation of Chronic Pain: A Safe Minimally Invasive, Effective Treatment Option
}

\author{
Laura Tyler Perryman* \\ Stimwave Technologies Incorporated, USA
}

Received: May 18, 2018; Published: May 29, 2018

*Corresponding author: Laura Tyler Perryman, Stimwave Technologies, Inc. 1310 Park Central Blvd South, Pompano Beach, Florida 33064, USA

\begin{abstract}
Nearly 90 million Americans suffer from chronic back pain and eventually get habituated to opioid pain medication hoping for long term relief. Spinal cord stimulation (SCS) evolved over 4 decades as an alternative and effective pain management therapeutic modality, especially in failed back surgery syndrome (FBSS). However technology limitations due to the bulk of the equipment and the limited frequency utilized in the stimulation restricted SCS applications. Over the past few years several advancements in nanotechnology and innovative minimally invasive surgical techniques have shown promising results. They also have a potential for less tissue damage, reduced hospital time and health care costs.
\end{abstract}

Aim: In this article, we introduce a minimally invasive nanotechnology wireless SCS system that has a wide range of frequency of stimulation and wider applications in pain management.

Material: We introduce the basis for nanotechnology stimulation and the preliminary experience with wider applications of wireless Neuromodulation for the relief of chronic pain in multiple clinical trials and case illustrations.

Results: The equipment utilizes a miniature stimulator device with microelectronics, percutaneously placed at the appropriate stimulation target. This is controlled by a wireless extracorporeal power generator to provide the required stimulation as moderated by the clinician and the patient. This wireless device has been so far clinically used in several chronic pain conditions like FBSS, facial pain, chronic regional pain syndrome and post herpetic neuralgia. Results have been very encouraging and devoid of complications related to implantable power generator (IPG) and its accessories. Few migrations occurred in a small number of patients and this has been the only possible complication of this technique indicating further refinements of the technology.

Conclusion: The nanotechnology and wireless devices have reduced the bulk of the implantable SCS equipment to a single electrode (with embedded sensors) due to the present day improved neural-electric interface (in place of brain-machine interface). Thus, they have become minimally invasive with less tissue trauma, fewer hospital hours, reduced hospital visits and better cosmetic results. Further studies with more evaluation metrics in larger groups of studies, however, are required for wider acceptance.

Keywords: Neuromodulation; Spinal Cord Stimulation; Peripheral Nerve Stimulation; Wireless Pain Relief; Minimally Invasive

Abbreviations: IPG: Implantable Power Generator; SCS: Spinal Cord Stimulation; FBSS: Failed Back Surgery Syndrome; WPG: Wireless Power Generator; GHz: Giga Hertz frequencies; DRG: Dorsal Root Ganglion; RF: Radiofrequency

\section{Introduction}

Spinal cord stimulation (SCS) is an established treatment modality for pain secondary to FBSS and is currently well accepted in terms of safety and efficacy as the number of Americans suffering from chronic back pain has been on the rise with increasing opioid habituation [1-5]. Although studies so far established the cost-ef fective benefits of SCS for FBSS, the traditional SCS has limitations that prohibit nearly $50 \%$ of patients from proceeding to long-term utilization [3-5]. Some of the reasons for the reported poor results include electrode migration, ineffective stimulation, device/IPG failure, and positional changes of the hardware. With the advent of nonomaterials and technology several improvements in the SCS 
equipment ensued to mitigate the complications and reduce the adverse events associated with the earlier devices, thus paving ways to increase the acceptance of the therapy thereby widening the spectrum of indications [6]. Minimally invasive surgical techniques, refined anchoring methods, reduced size of the IPG with increased life span of the battery and wireless transfer of power are some of these recent advances.

\section{Wireless Neuromodulation with Nanotechnology}

Traditional SCS equipment comprises of implantable electrodes enclosed inside a catheter, long extension wires connecting these electrodes to an IPG. All these components are placed surgically inside the patient body and complications related to the surgical (multiple) procedures as well as the failures of any of these components are by default become those of the Neuromodulation therapy. Several efforts in the industry perspective intend to reduce the bulk with improved efficiency; mostly IPG related durability and extension of life of the battery. Some of them have been successful but still surgical placement is required for all the components to work, including the long segment tunneling to connect the IPG with the stimulating leads.

Advancement in this field is the new external wireless power generator (WPG) that applies a dipole antenna for electric field coupling. This is accomplished via 'microwaves' (very short-length pulsed electromagnetic (EM) waves) at Giga Hertz frequencies (GHz). This wireless device (currently from Stimwave technologies), instead of lower inductive frequencies (ranging between $100-500 \mathrm{kHz}$ ) for most of the implanted medical devices, is powered by Radiative electric field coupling through tissue at microwave frequencies. These waves enable miniature sized implants to be placed significantly deep in tissue through a needle and yet accessible. The higher frequencies applied afford minimal power loss and offer superior energy transfer to smaller sized implants [7]. This energy transfer phenomenon was mentioned by Feynman, Father of Nanotechnology, as the principle behind the frequency vs wavelength changes in his talk on nanotechnology (there is plenty of room at the bottom) and accordingly skin depth only decreases with square root of the scale ratio (scale on which frequency goes up and wavelength comes down). As he rightly mentioned, superconductors today have reduced the resistance in modern physics [8].

The nonmaterial implant in the WPG is capable of delivering the clinically appropriate range of stimulation at 800-1350 um diameter, a very small sized implant compared to the conventional SCS-IPG. Additionally, the amperage requirements for dorsal root ganglion (DRG) stimulation compared to SCS are much lower thus increasing the longevity of the WPG. The implant size is equivalent to the standard lead body of the SCS and incorporates the Nano electronics within itself. It can be included in to different contact types of leads ( 4 or 8 contacts) suitable for both percutaneous cylinder type or paddle type electrodes. The receiver wire is mated to the implant to communicate with the external/wireless power generator (Figure 1).

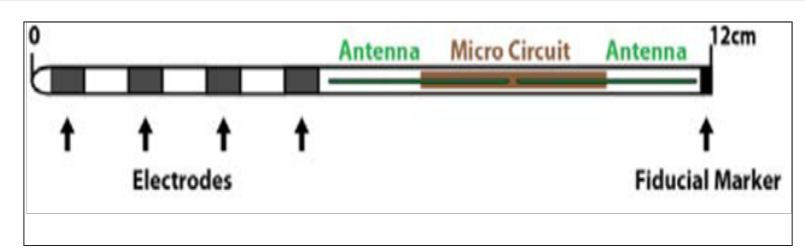

Figure 1: Neuro-stimulator electrode, MRI compatible, for both 1.5 and 3 Tesla.

An oscillating electric field is created as the dipole antenna receiver intercepts the microwave EM frequencies emanated from the EPG. The antenna within the device can vary between $2 \mathrm{~cm}$ and $8 \mathrm{~cm}$ in length (with modifications possible depending upon the depth of implantation). The EM energy can be dissipated at variable depths starting from skin to bone across the intervening fat, muscle, blood vessels. Previous experimental models demonstrated that frequency at $\mathrm{GHz}$ range were more energy efficient [9]. The animal models showed that deeper placements require longer antenna to receive the require power. As part of an application specific integrated circuit, each contact on the stimulating lead is provided with exclusive power capabilities, since the circuits inside the contacts produce very specific charge balanced waveforms (Figure 2).

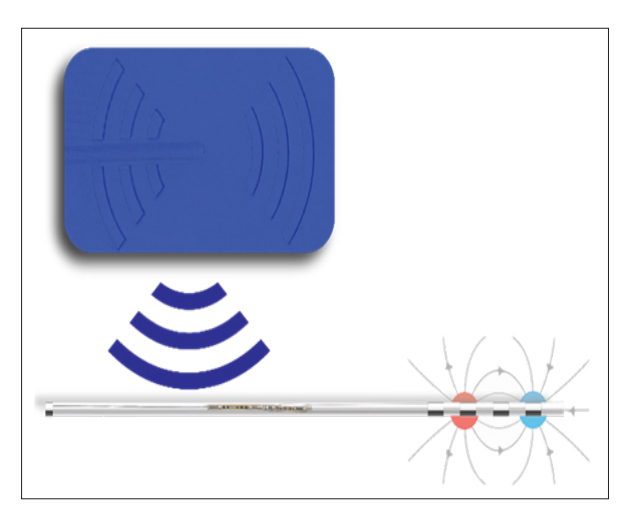

Figure 2: Neurostimulator receiver. The contacts on the electrodes are managed by independently integrated, circuits that are application specific. The circuitry system within the device produces charge-balanced waveforms.

\section{The WPG In Place of Implantable Power Generators}

Just like the present day cellular phones, WPG employs similar transfer technology. The average pulse output of power is up to 1 Watt depending upon the required stimulation and the depth at which the stimulator is placed. The WPG has a radiofrequency (RF) transmitter that transforms the stimulation waveforms in to a signal as per the program setting given by the clinician or the patient; while a microprocessor within the transmitter regulates the settings and data transfers (Figure 3). A controller utilizing Bluetooth technology makes it convenient for both the patient and the clinician to access the WPG for suitable modifications; which can also be performed via an app on a cellular phone [7]. Additionally, neuronal damage is very less likely with EM wave forms at micro wavelengths; since high frequency does not activate the cell membranes. Thus, the wireless nanotechnology device not only affords 
minimally invasive surgical implants but the microwave energy is more compatible with biological safety also.

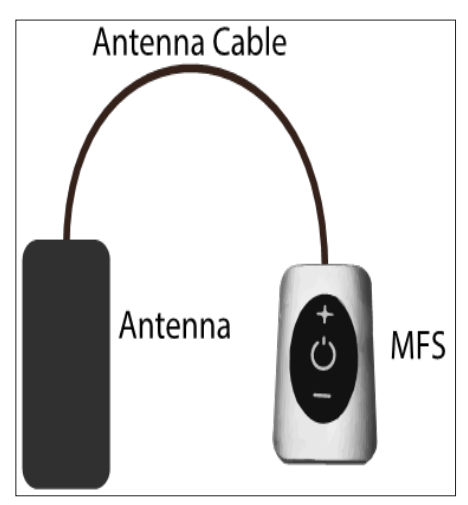

Figure 3: Freedom (Stimwave) SCS external device.

\section{Discussion}

SCS, utilized for over 40 years, has been proven to be cost effective therapy for chronic pain due to failed back surgery, chronic regional pain syndromes (CRPS) and neuralgias [10-14]. However, SCS is not without limitations and complications in its conventional form that employs all implantable components; electrodes, connecting wires and IPG $[2,13,14]$. A significant number of patients also fail to proceed beyond the trial stimulation, in as much as $50 \%$ losing the benefits of long term SCS advantages [2-5]. Additional equipment related complications like lead migration, painful surgical sites, and infection and IPG failures complicate the traditional approach. In some patients, SCS cannot access the anatomical location of pain $[2,14]$.

To reduce the complications associated with the bulk of the conventional SCS equipment, many modifications have been proposed. The most important upcoming innovation is wireless access to the implant to provide Neuromodulation. In several clinical conditions, wireless nanotechnology stimulation system has been utilized for SCS, DRGS and PNS throughout Europe and in the USA over the past couple of years with encouraging responses, albeit in case studies.

The operating capabilities of wireless power transmission in biological media have been demonstrated at $\mathrm{GHz}$ range (as against the $\mathrm{MHz}$ of the conventional stimulation methods), by Poon et al offering potential advantages $[9,15]$. There was a remarkable reduction in the size of the receiver at this frequency. Subsequent studies by Tyler Perryman et al demonstrated the relationships between tissue depth and energy transmission in animal models $[15,16]$ The author conducted experimental studies in pig models to verify the tissue depth and the accessibility of the wireless transmission of signals to achieve effective current density [16]. In this study, at $915 \mathrm{MHz}$, the dipole antenna of the WPG could energize the nanostimulators placed at the depth of $12 \mathrm{~cm}$ in porcine models; an antenna of $4.3 \mathrm{~cm}$ was more efficient.

In clinical scenario, successful wireless stimulation and significant pain relief was observed in patients with back pain, leg pain, neuralgia following herpes zoster, craniofacial pain, occipital neuralgia, and complex regional pain syndrome [17-20]. Adverse events or complications in these short case series and reports were minimal. The wireless access by the WPG, to the nanoelectode required implantation of the stimulating electrode (with embedded sensors) only; excluding additional surgical trauma from implantation of IPG and its accessories. Thus, complications related to these components were avoided. There was reduced surgical trauma, operating time, usage of consumables with increased comfort and cosmetic result to the patient.

\section{Summary}

SCS, although an effective therapeutic modality for chronic pain disorders, in its conventional implantable equipment design has significant complications and failures. Technological advancements and surgical refinements to reduce these adverse events resulted in minimally invasive techniques and implants. Wireless navigation and better understanding of nanotechnology provided refinements to the equipment reducing the tissue trauma, number of surgical procedures, operating time and in turn, health care costs in limited number of case studies. Stimwave technologies have improved upon these developments to provide better clinical outcomes and several clinical studies are being conducted in large numbers across the USA and Europe utilizing numerous instruments to measure the outcomes as well as adverse events.

\section{References}

1. Praeger J (2010) Estimates of annual spinal cord stimulator implant rises in the United States. Neuromodulation 13: 68-69.

2. Cameron $\mathrm{T}$ (2004) Safety and efficacy of spinal cord stimulation for the treatment of chronic pain: a 20-year literature review. Journal of Neurosurgery 100: 254-267.

3. Kumar K, Taylor RS, Jacques L, Milbouw G, Buchser E, et al. (2008) The effects of spinal cord stimulation in chronic pain are sustained: a 24-month follow-up of the prospective randomized controlled multicenter trial of the effectiveness of spinal cord stimulation. Neurosurgery 63: 762-770.

4. Kumar K, Hunter G, Demeria D (2006) Spinal cord stimulation in treatment of chronic benign pain: challenges in treatment planning and present status, a 22-year experience. Neurosurgery 58: 481-496.

5. North RB, Kidd DH, Farrokhi F, Piantadosi SA (2005) Spinal cord stimulation versus repeated lumbosacral spine surgery for chronic pain: a randomized, controlled trial. Neurosurgery 56: 98-107.

6. Slavin KV (2014) Spinal stimulations for pain: future applications Neurotherapeutics 11: 535-542.

7. Yearwood TL, Perryman LT (2016) Peripheral neurostimulation with a microsize wireless stimulator. In. Slavin (Ed) Stimulation of the peripheral nervous system. The Neuromodulation Frontier. Prog Neurol Surg Basel Karger 29: 168-191.

8. Feynman RP (1959) there's plenty of room at the bottom. Presentation to the American Physical Society.

9. Poon AS, O’Driscoll S, Meng TH (2007) Optimal operating frequency in wireless power transmission for implantable devices. Conf Proc IEEE Eng Med Biol Soc 2007: 5674-5679.

10. Kapural L, Yu C, Doust M, Morgan DM, Brown LL, et al. (2015) Novel 10$\mathrm{kHz}$ high-frequency therapy (HF10 therapy) is superior to traditional low-frequency spinal cord stimulation for the treatment of chronic back and leg pain the SENZA-RCT randomized controlled trial. Anesthesiology 123: 851-860. 
11. Deer TR, Mekhail N, Provenzano D (2014) The appropriate use of neurostimulation of the spinal cord and peripheral nervous system for the treatment of chronic pain and ischemic diseases: the Neuromodulation Appropriateness Consensus Committee. Neuromodulation 17: 515-550.

12. Mekhail NA, Aeschbach A, Stanton Hicks M (2004) Cost benefit analysis of neurostimulation for chronic pain. Clin J Pain 20: 462-468.

13. Turner JA, Loeser JD, Deyo RA, Sanders SB (2004) Spinal cord stimulation for patients with failed back surgery syndrome or complex regional pain syndrome: a systematic review of effectiveness and complications. Pain 108: $137-147$.

14. Mekhail NA, Mathews M, Nageeb F, Guirguis M, et al. (2011) Retrospective review of 707 cases of spinal cord stimulation: indications and complications. Pain Pract 11: 148-153.

15. Poon A, O’Driscoll, Meng TH (2010) Optimal frequency for wireless power transmission in to dispersive tissue. IEEE Trans Antennas Propag 58: 1739-1750.

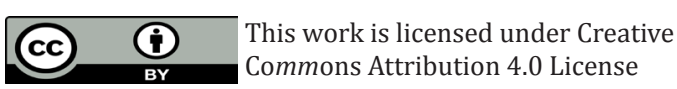

Submission Link: https://biomedres.us/submit-manuscript.php
16. Tyler Perryman L, Larson P, Glaser J (2016) Tissue depth study for a fully implantable, remotely powered and programmable wireless neural stimulator. Int J Nano Stud Technol S2: 1-6.

17. Billet B, Wynendaele R, Vanquathem N (2017) A novel minimally invasive wireless technology for neuromodulation via percutaneous intercostal nerve stimulation (PNS) for post-herpetic neuralgia: A case report with short term follow up. Pain Pract 18: 374-379.

18. Weiner RL, Garcia CM, Vanquathem N (2017) A novel miniature wireless neurostimulator in the management of chronic craniofacial pain: preliminary results from a prospective pilot study. Scand J Pain17: 350354.

19. Perryman LT, Speck B, Weiner RL (2017) A novel wireless minimally invasive neuromodulation device for the treatment of chronic intractable occipital neuralgia: case illustrations. J Neurol Stroke 6: 00213.

20. Herschkowitz D, Kubias J (2018) Wireless peripheral nerve stimulation for complex regional pain syndrome type I of the upper extremity: a case illustration introducing a novel technology. Scan J Pain.

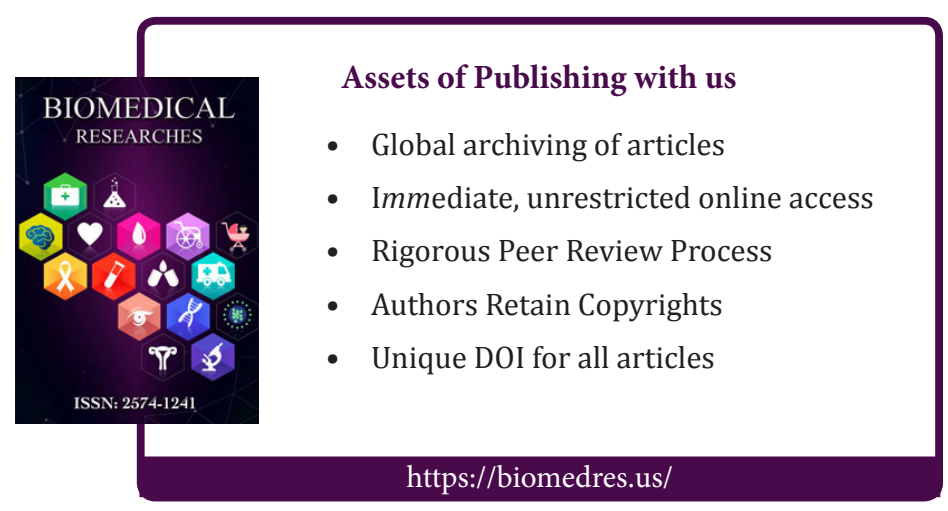

